\title{
Critical adjustments in a department of orthopaedics through the COVID-19 pandemic
}

\author{
Gonzalo Luengo-Alonso ${ }^{1}$ (D) - Fernando García-Seisdedos Pérez-Tabernero ${ }^{1}$ (i) • Miguel Tovar-Bazaga ${ }^{1}$ (D) \\ José Manuel Arguello-Cuenca ${ }^{1}$ (ID $\cdot$ Emilio Calvo $^{1}$
}

Received: 15 May 2020 / Accepted: 18 May 2020 / Published online: 30 May 2020

(C) SICOT aisbl 2020

\begin{abstract}
Purpose SARS-CoV-2's new scenario has forced health systems to work under extreme stress urging to perform a complete reorganization of the way our means and activities were organized. The orthopaedic and trauma units have rescheduled their activities to help SARS-CoV-2 units, but trauma patients require also treatment, and no standardized protocols have been established.

Methods A single-centre cross-sectional study was performed in a tertiary hospital. Two different periods of time were analyzed: a two week period of time in March 2019 (pre-SARS-CoV-2) and the same period in March 2020 (SARS-CoV-2 pandemic time). Outpatient's data, emergency activity, surgical procedures, and admissions were evaluated. Surgeons' and patient's opinion was also evaluated using a survey.

Results A total of $\sim 16 \mathrm{k}(15,953)$ patients were evaluated. Scheduled clinical appointments decreased by $\sim 22 \%$. Urgent consultations and discharge from clinics also descended ( $\sim 37 \%$ and $\sim 20 \%$ respectively). Telemedicine was used in $90 \%$ of outpatient clinical evaluations. No elective surgical procedures during SARS-CoV-2 time were scheduled, and subtracting the effect of elective surgeries, there was a reduction of inpatient surgeries, from $\sim 85 \%$ to $\sim 59 \%$. Patients delayed trauma assistance more than 48 hours in 13 cases (35\%). Pre-operative admission for hip fractures decreased in ten hours on average. Finally, surveys stated that patients were more in favour than surgeons were to this new way to evaluate orthopaedic and trauma patients based strongly on telemedicine. Conclusion Detailed protocols should be standardized for surgical departments during the pandemic. This paper offers a general view in how this virus affects an orthopaedic unit and could serve as a protocol and example for orthopaedic and trauma units. Even in the worst scenario, an orthopaedic and trauma unit could offer an effective, efficient, and quality service. SARS-CoV-2 will set up a new paradigm for health care in orthopaedics and trauma.
\end{abstract}

Keywords SARS-CoV-2 $\cdot$ COVID-19 $\cdot$ Telemedicine pandemic $\cdot$ Orthopaedic and trauma $\cdot$ Surgery

\section{Introduction}

The pandemic caused by the previously unknown SARSCoV-2 (2019-nCoV, COVID-19) virus was first detected in Wuhan, China [1-3]. This new scenario has forced health systems to work under extreme stress, using limited resources

Emilio Calvo

ecalvo@fjd.es

1 Department of Orthopedic Surgery and Traumatology, Hospital Universitario Fundación Jiménez Díaz, IIS Fundación Jiménez Díaz, Universidad Autónoma, Avda. Reyes Católicos, 2, 28040 Madrid, Spain and be put through a quick and complete reorganization of the way our means and activities were organized [4-6].

High mortality rates and intensive care unit (ICU) patients have been a challenge to every single health system [7]. Incidences of infected population and mortality are updated daily by the World Health Organization. To date, a total of 231 countries have reported cases, with a number close to 1.6 million confirmed diagnosis, and almost $\sim 100 \mathrm{k}$ deaths all over the world [8]. Spain was one of the first countries after China to be hit with this epidemic, with its capital, Madrid, being the hardest hit region. Spain has seen a total of $\sim 220 \mathrm{k}$ SARS-CoV-2 cases (3.6k per million people), with $\sim 20 \mathrm{k}$ deaths (374 per million) [9].

Global experience covering how to adapt existing health systems to this new scenario is really limited. Few 
publications have reported alternative methodologies to adapt the operations and processes of a surgical department to this devastating scenario $[10,11]$. SARS-CoV-2 has required orthopaedics and traumatology departments to reschedule daily activities, despite not being in the frontline fighting this virus. Our orthopaedic and trauma activity had to be modified dramatically in order to collaborate directly with ICU and other medical units, to support the daily care of patients with 2019$\mathrm{nCoV}$ disease and to provide our best health care delivery in a new scenario where the Spanish government sets strict confinement measures.

While there has been a shift in the orthopaedics and traumatology departments' capacity towards SARS-CoV-2, the team has continued to serve patients requiring urgent care. Procedures such as trauma, tumours, and surgical complications (infections) were performed even during epidemic time. This exceptional situation obliged us to develop new protocols to preserve the safety of both patients and orthopedic surgeons [12-14].

The aim of our study is to share our experience and new strategies in the SARS-CoV-2 epidemics. This report provides great insight and useful and actionable learning to help orthopaedic and trauma departments adapt to an epidemic such as SARS-CoV-2 in an efficient way.

Our hypotheses are as follows: (1) patients evaluated in clinics and emergency room (ER) will decrease due to the lockdown associated to the pandemics, (2) discharges from consultation in orthopaedic and trauma units will increase as result of patient's fear to SARS-CoV-2, and (3) the number of surgical procedures will decline, but the average in-hospital stay of patients undergoing emergent surgery will decrease.

\section{Materials and methods}

\section{Study design}

Single-center cross-sectional study (observational, nonrandomized at a specific point in time) was performed in two different periods of time: pre-epidemic (pre-SARSCOV-2) and intra-epidemic (during SARS-COV-2 time) to compare these two very different scenarios. The subjects that inform the study were consultant orthopaedic surgeons and orthopaedic and trauma patients treated at our hospital in our various areas: outpatients, inpatients, ER, and operating room.

\section{Inclusion/exclusion criteria}

Inclusion criteria: Orthopaedic and trauma patients evaluated and treated by our service staff during a period of two weeks in two different points in time, pre-epidemic, and intraepidemic.
Exclusion criteria: We excluded patients 18 years old and younger as paediatric health care was centralized in two centres in Madrid during the intra-epidemic period.

\section{Study development and groups}

Patients were divided into two groups. The first one includes patients evaluated and treated during the third and fourth week of March 2019 (pre-epidemic). The second group includes patients evaluated and treated during the third and fourth week of March 2020 (intra-epidemic).

\section{Data analyzed}

Data used in this study was collected anonymously from our hospital database. We analyzed the following variables in both groups:

\section{Outpatients data}

Gathered data related to the number of patients evaluated in person and telematically, either through phone call or realtime videoconferences, main reason for consulting, and subsequent appointments resulting from the visit were recorded regarding the following variables: overall number of patients evaluated due to orthopaedic or trauma pathology, main reason for consulting, number of specific subspecialty appointments (i.e., shoulder/elbow, hand/microsurgery, hip, knee, foot/ankle, spine and general trauma) drawn from these evaluations, and categorized as urgent or standard appointments, and number of discharged patients from clinics.

\section{Emergency room}

Overall ER department attendance; time elapsed from injury; and specific complaint classified as hand, shoulder/elbow, hip, and other lower extremity as well as specific injury were recorded.

3. Surgical procedures

Number, type of procedures, average in-hospital stay, and inpatient/outpatient ratio were assessed. Surgical procedures were allocated into four categories: trauma, infection, tumours, and non-trauma/elective surgery.

4. Inpatients and outpatients.

The surgical procedures performed were distributed into four categories: trauma, infection, tumours, and non-trauma/ elective surgery. We also looked at total and pre-operative average stay for admitted patients. 


\section{Perceived quality}

Patient and surgeon's perceived quality of medical care delivery was evaluated through this epidemic period using an anonymous survey carried out following a specifically designed questionnaire over the phone. Items were scored using a numerical rating scale (NRS) ranking 1 to 10 . Patients and surgeons were also asked about their perception on the possibility that a similar system of telemedicine might be followed for future health care delivery in outpatient clinics (Tables 1 and 2). Sixteen orthopedic surgeons and 300 patients were randomly selected for this purpose. A balanced number of subgroups including all anatomic areas was procured.

\section{Quality appraisal}

The quality of the study was assessed using STROBE system [15]. Out of 22 possible items, we used 18 of the STROBE checklist for the methodological assessment (Table 3).

\section{Statistical analysis}

Relevant data was inserted into an electronic database (Microsoft ${ }^{\circledR}$ Excel for Windows ${ }^{\circledR}$ (Microsoft Corp, Redmond, WA)) for further analysis. Mean and standard deviation was used to describe quantitative variables, and frequency and percentage for qualitative variables. Data analysis was carried out using IBM SPSS version 24.0 (IBM SPSS,
Armonk, NY, USA), and significance of pooled estimates was set at $p<0.05$.

Institutional review board approval under act 07/20 was obtained prior to the commencement of the study and informed consent was obtained from all patients.

\section{Results}

A total of $\sim 16 \mathrm{k}$ (15.953) patients from Group 1 (pre-SARSCoV-2) and Group 2 (SARS-CoV-2) were analyzed, following this distribution: $97.8 \%$ clinical consultations (15.607) and $2.2 \%$ surgical procedures (346) (Table 4).

Scheduled clinical consultations decreased severely by $22 \%$. General outpatient clinic attendances showed a relative decline of $\sim 16 \%$, but ER attendances experienced a dramatic reduction of $\sim 37 \%$. Discharge from outpatient clinics also decreased from 1331 to 837 in SARS-CoV-2 group, which represents a relative reduction of $\sim 20 \%$ (Table 5).

Drilling deeper into consultations, appointments scheduled from clinical consultation decreased in 759 consultations ( $26 \%$ ) when compared to Group 1. With regard to subspecialties, all showed a decrease which was more severe in hand/microsurgery $(\sim 12 \%)$ and spine $(\sim 73 \%)$, while knee consultations increased by $\sim 27 \%$ (Table 6 ).

During the SARS-CoV-2 epidemic, less than $10 \%$ of outpatient clinical appointments were performed in person, while the rest of them were done virtually.

Table 1 Telematics surveys to patients

- Telematics Surveys to patients:

1. Rate telematics consultation from 0 to 10.0 (very unsatisfied) - 10 (very satisfied) ( )

2. Rate information received from your surgeon 0 to 10.0 (very unsatisfied) 10 (very satisfied) ()

3. Did your doctor dedicated enough time during your telematic consultation to evaluate your case?

No () Yes ()

4. Are you satisfied with telematics evaluation?

No () Yes ()

5. Would you prefer face-to-face evaluation?

No () Yes ()

6. Do you think that telematic follow-up would be appropriate for some of your upcoming consultations?

No ( ) Yes ()

7. Would you prefer videoconference instead of phone calls?

No () Yes ()

- Epidemiology data:

- Male () Age : 18-44 ( ) 45-64 ( ) >65( )

- Female () Age : 18-44( ) 45-64( ) >65( ) 
Table 2 Orthopedic surgeon survey

\section{- Orthopedic Surgeon Survey}

8. Rate consultation from 0 to 10.0 (very unsatisfied) -10 (very satisfied)

( )

9. Would you prefer a face-to-face evaluation?

No ( ) Yes ( )

10. Do you think a face-to-face consultation would provide more information or change your medical attitude?

No ( ) Yes ( )

11. From now on, do you think a telephone follow-up would be appropriate some of the upcoming evaluations?

No ( ) Yes ( )

12. What percentage do you think you could evaluate using telemedicine without losing care quality?

( ) $\%$

Table 3 STROBE item used

\begin{tabular}{|c|c|}
\hline Item & Description \\
\hline \multirow[t]{2}{*}{ Title and abstract (1) } & (a) Indicate the study's design with a commonly used term in the title or the abstract \\
\hline & (b) Provide in the abstract an informative and balanced summary of what was done and what was found \\
\hline \multicolumn{2}{|l|}{ Introduction } \\
\hline $\begin{array}{l}\text { Background/rationale } \\
\text { (2) }\end{array}$ & Explain the scientific background and rationale for the investigation being reported \\
\hline Objectives (3) & State specific objectives, including any prespecified hypotheses \\
\hline \multicolumn{2}{|l|}{ Methods } \\
\hline Study design (4) & Present key elements of study design early in the paper \\
\hline Setting (5) & Describe the setting, locations, and relevant dates, including periods of recruitment, exposure, and data collection \\
\hline Participant (6) & Give the eligibility criteria, and the sources and methods of selection of participants. \\
\hline Variables (7) & Clearly define all outcomes, predictors, potential confounders, and effect modifiers. Give diagnostic criteria, if applicable \\
\hline $\begin{array}{l}\text { Data } \\
\text { source/measurements } \\
(8)\end{array}$ & $\begin{array}{l}\text { For each variable of interest, give sources of data and details of methods of assessment (measurement). Describe } \\
\text { comparability of assessment methods if there is more than one group. }\end{array}$ \\
\hline $\begin{array}{l}\text { Quantitative variables } \\
\text { (11) }\end{array}$ & $\begin{array}{l}\text { Explain how quantitative variables were handled in the analyses. If applicable, describe which groupings were chosen and } \\
\text { why. }\end{array}$ \\
\hline \multirow[t]{3}{*}{ Statistical methods (12) } & (a) Describe all statistical methods, explain how missing data were addressed if applicable \\
\hline & (b) Describe any methods used to examine subgroups and interactions \\
\hline & (c) Explain how missing data were addressed \\
\hline \multicolumn{2}{|l|}{ Result } \\
\hline Participants (13) & $\begin{array}{l}\text { Report numbers of individuals at each stage of study - e.g., numbers potentially eligible, examined for eligibility, confirmed } \\
\text { eligible, included in the study, completing follow-up, and analyzed }\end{array}$ \\
\hline Descriptive data (14) & Indicate number of participants with missing data for each variable of interest \\
\hline Outcome data (15) & Report numbers of outcome events or summary measures over time. \\
\hline Main results (16) & Give unadjusted estimates and, if applicable, confounder-adjusted estimates and their precision \\
\hline \multicolumn{2}{|l|}{ Discussion } \\
\hline Key results (18) & Summarize key results with reference to study objectives \\
\hline Limitations (19) & $\begin{array}{l}\text { Discuss limitations of the study, taking into account sources of potential bias or imprecision. Discuss both direction and } \\
\text { magnitude of any potential bias }\end{array}$ \\
\hline Interpretation (20) & $\begin{array}{l}\text { Give a cautious overall interpretation of results considering objectives, limitations, multiplicity of analyses, results from } \\
\text { similar studies, and other relevant evidence }\end{array}$ \\
\hline Generalisability (21) & Discuss the generalizability (external validity) of the study results \\
\hline
\end{tabular}


Table 4 Group 1: patients pre-SARS-CoV-2 (2019). Group 2: patients SARS-CoV-2 (2020)

\begin{tabular}{lll}
\hline & Clinical Evaluation & Surgical procedures \\
\hline Group 1 & 8760 & 304 \\
Group 2 & 6847 & 42 \\
Total & 15,607 & 346 \\
\hline
\end{tabular}

Concerning surgical activity, the number of surgical procedures declined dramatically in the two week period under analysis from 304 surgical cases in 2019 to 34 in 2020 , representing an $88.8 \%$ drop-off. This decrease was heavily driven by elective surgery that were not scheduled during the epidemic time, whereas a total of 219 procedures were recorded in Group 1. Even after taking out elective surgeries from the sample, a large drop of $60 \%$ in non-trauma/elective surgery could be detected, from 85 in Group 1 to 34 in Group 2. Also, subtracting the effect of elective surgery, a reduction of inpatient surgery, from $\sim 85$ to $\sim 59 \%$ (a relative drop of $\sim 31 \%$ ), was observed trying to keep these patients away of the pandemic as soon as possible. Similarly, pre-operative average in hospital stay for hip fractures in Group 1 was more than 30 hours compared to a mean time of 20 hours in Group 2. Mean admission time for hip fractures in Group 1 was seven days and seven hours (6 days postoperatively) compared to a mean time of two days mean and one day and three hours post-operatively, in Group 2 (Table 7). Trauma cases decreased more than 54\% during epidemic time compared to the previous year demonstrating the effect of lockdown. Shoulder/elbow and hip fractures showed the biggest drops with $\sim 68 \%$ and $57 \%$ declines respectively (Table 8 ).

Concerning urgent trauma, patients were reluctant to attend the ER. In pre-SARS-COV-2, patient's delay was an exceptional $\sim 5 \%$, opposite to what happened in SARS-COV-2 crisis, in which this percentage increased to $\sim 35 \%$. Some patients requested medical attention even later than a week $(16.6 \%)$ suffering serious injuries (i.e., hip fractures) (Table 9).

Table 5 Clinical appointments

\begin{tabular}{lll}
\hline & $\begin{array}{l}\text { Group 1 } \\
\text { (pre-SARS-CoV-2) }\end{array}$ & $\begin{array}{l}\text { Group 2 } \\
\text { (SARS-CoV-2) }\end{array}$ \\
\hline Total clinical appointments & 8760 & 7831 \\
Attendance & $7831(89.39 \%)$ & $5134(74.98 \%)$ \\
Urgent appointments & $680(7.76 \%)$ & $334(4.87 \%)$ \\
Non-contact & $929(10.60 \%)$ & $1713(25.01 \%)$ \\
Discharges & $1331(15.19 \%)$ & $837(12.22 \%)$ \\
\hline
\end{tabular}

In terms of satisfaction, surgeon's perceived quality of medical care delivery reached an overall score of 6.6 points. As for the effectiveness of telemedicine clinical evaluations, surgeons believed that they could conduct up to $\sim 25 \%$ virtually (Table 10). As for surgeon views, most of them ranked telemedicine as the preferred method during SARS-COV-2, even though a majority agreed a physical consultation would provide an additional value to telemedicine. In terms of remote consultation, the surgeon's opinion was divided whether to continue with telemedicine or not after the pandemic (Table 10).

On the contrary, patients rated the new system for telematic health care delivery with higher scores both regarding the new consultation system and the information received (Table 11). However, a vast majority of patients would have preferred a face-to-face interview, and only half of them would consider following the same system after the pandemic.

\section{Discussion}

SARS-CoV-2 stretched health systems all over the world [5, $6,10,13]$. Since the first diagnosis in China, nearly 2 million cases have been confirmed worldwide, 1 million of those in Europe. Within Europe, Spain is one of the countries with the highest number of confirmed cases, $\sim 200 \mathrm{k}$, and Madrid has been the hardest-hit city, with $\sim 51 \mathrm{k}$ cases up to date. Although experts and data are encouraging, the pandemic continues rising, with nearly 400 deaths daily and a total of $\sim 20 \mathrm{k}$ deaths in our country [9].

The fact that Madrid has been one of the first big European cities hit by the pandemic has allowed us to share with the orthopedic community our experience in managing orthopaedic health care delivery in such difficult conditions. In this scenario, we have contributed supporting those medical units directly involved in the treatment of patients affected by SARS-CoV-2, but we also continued providing healthcare to our patients. We have therefore applied a cross-sectional observational study, evaluating data from specific points in time, allowing us to compare our two very different scenarios, preSARS-CoV-2 vs SARS-CoV-2. Results from this type of studies are more generalizable to geographically defined populations, as in this case is redefining orthopaedic activity in SARS-CoV-2 pandemic time. To our knowledge, this is the first study reporting fast changes experienced by a large orthopaedic department in an academic hospital as result of the SARS-CoV-2 pandemic, and several interesting conclusions can be drawn from this experience.

Attendance in all working areas (ER, surgery, consultation, and admission) decreased as expected as consequence of SARS-CoV-2. Only patients requiring urgent care, such as trauma, tumours, and infections have been scheduled as 
Table 6 Appointments made in clinic consultation

\begin{tabular}{llll}
\hline & & Group 1 (pre-SARS-COV-2) & Group 2 (SARS-COV-2) \\
\hline Foot and ankle & Urgent & 5 & 2 \\
& Total & 434 & 354 \\
Knee & Urgent & 18 & 1 \\
& Total & 509 & 478 \\
Hip & Urgent & 5 & 0 \\
Spine & Total & 163 & 142 \\
& Urgent & 45 & 9 \\
Hand/microsurgery & Total & 720 & 530 \\
Shoulder/elbow & Urgent & 29 & 2 \\
& Total & 545 & 358 \\
Total & Urgent & 12 & 5 \\
\end{tabular}

expected. Admissions were also affected, as procedures were performed on an outpatient basis whenever possible, but preoperative and overall average stay of those cases managed inpatient decreased dramatically. It is interesting to note that this finding was strongly influenced by management of hip fractures. Hip fracture is a common procedure that cannot be delayed and has an important influence on the average inhospital stay. Interestingly, average stay dropped sharply from seven days in the same period of March 2019 to less than two days during the pandemic. This reduction was mostly due to a sharp minimization of post-operative stay. Even in-hospital admission time for a hip fracture was in many cases less than 24 hours long. This represents a new scenario, as we do not know what will be the outcome of these fragile patients without post-operative orthogeriatric care. As for the long-term persistence of this shift from inpatient to outpatient treatments, it is hard to predict. We have noticed families played a key role in discharging patients as soon as possible due to the risk of SARS-CoV-2 infection, but it is yet to demonstrate that that

Table 7 Surgical procedures comparison

\begin{tabular}{lccccc}
\hline & \multicolumn{2}{c}{ Group 1 (pre-SARS-COV-2) } & & \multicolumn{2}{l}{ Group 2 (SARS-COV-2) } \\
\cline { 2 - 3 } \cline { 5 - 6 } \cline { 5 - 6 } & Outpatients & Inpatients & & Outpatients & Inpatients \\
\hline Elective & 164 & 55 & & 0 & 0 \\
Trauma & 7 & 10 & & 14 & 2 \\
Hip fractures & 0 & 28 & & 0 & 12 \\
Infection & 0 & 11 & & 0 & 1 \\
Tumors & 0 & 5 & & 0 & 3 \\
Spine & 6 & 18 & & 0 & 2 \\
Total & 177 & 127 & & 14 & 20 \\
\hline
\end{tabular}

level of engagement and support will be sustained after the pandemic.

At the ER, a dramatic decrease (more than 90\%) in patients requiring orthopedic evaluation was also observed. This decrease was driven by both the confinement measurements and by patients being afraid of SARS-CoV-2. It is important to point out that the majority of patients who attended the ER several days after the traumatic event occurred probably due to fear of infection transmission of coronavirus, even in the case of hip fractures. Therefore, delayed treatment of trauma cases should be expected in countries affected by the pandemic.

Evaluation in outpatient clinics changed to telemedicine assistance in the vast majority of cases, maintaining in-person evaluation only for those cases in which physical evaluation or direct cast or wound care was deemed necessary. This has led to two unprecedented changes: a notable transformation in the doctor-patient relationship and also a significant impact in surgeon's practice. Accordingly, surveys were performed to orthopaedic surgeons and patients aimed to assess the perceived quality of this appraisal. Overall, orthopaedic surgeon

Table 8 Trauma cases attended

\begin{tabular}{lll}
\hline & $\begin{array}{l}\text { Group 1 } \\
\text { (pre-SARS-COV-2) }\end{array}$ & $\begin{array}{l}\text { Group 2 } \\
\text { (SARS-COV-2) }\end{array}$ \\
\hline Hand/microsurgery & 14 & 8 \\
Shoulder and elbow & 19 & 6 \\
Lower extremity & 18 & 10 \\
Hip fractures & 28 & 12 \\
Total & 79 & 36 \\
\hline
\end{tabular}


Table 9 ED trauma cases

\begin{tabular}{lll}
\hline & $\begin{array}{l}\text { Group 1 } \\
\text { (pre-SARS-COV-2) }\end{array}$ & $\begin{array}{l}\text { Group 2 } \\
\text { (SARS-COV-2) }\end{array}$ \\
\hline Less than 48 h & 75 & 23 \\
More than 48 h & 3 & 7 \\
More than 7 days & 1 & 6 \\
Total & 79 & 36 \\
\hline
\end{tabular}

satisfaction on the quality of health care delivered reached fair result of 6.7 points. This critical SARS$\mathrm{CoV}-2$ period and a radical change in our daily practice could be the main reasons for these results. Results from our surgeons are quite rational, as the majority considered that telemedicine was very helpful in this special setting. However, they did not agree with the possibility of continuing this telemedicine system for patient consultation after the pandemic. This is probably due to the fact that the majority of telemedicine consultations corresponded to follow-up appointments of previously evaluated patients, but the system might have limitations for first clinical appointments. An interview differentiating first and follow-up appointments should be performed to clarify this point. The experience reported in this study provides a new insight in outpatient's clinics health care delivery and anticipate a new paradigm in the management of outpatient orthopedic

Table 10 Survey results after interviewing 16 orthopedic surgeons

\begin{tabular}{lll}
\hline & $\begin{array}{c}\text { Satisfaction } \\
(0-10)\end{array}$ & $\begin{array}{l}\text { Clinical evaluations } \\
\text { that could be done } \\
\text { telematically } \\
(0-100 \%)\end{array}$ \\
Knee & 7.5 & $35 \%$ \\
Hip & 8 & $30 \%$ \\
Shoulder/elbow & 7 & $25 \%$ \\
Spine & 6.5 & $15 \%$ \\
Hand/microsurgery & 5 & $22,5 \%$ \\
$\begin{array}{l}\text { Foot and ankle } \\
\text { Trauma }\end{array}$ & 6.5 & $22,5 \%$ \\
& 6.5 & $12,5 \%$ \\
2. Would you prefer a face-to-face & $81.25 \%$ & No \\
$\quad$ evaluation? & $18.75 \%$ \\
3. Do you think a face-to-face & $87.5 \%$ & $12.5 \%$ \\
$\begin{array}{l}\text { consultation would provide } \\
\text { more information or change }\end{array}$ & & \\
$\begin{array}{l}\text { your medical attitude? } \\
\text { 4. From now on, do you think a } \\
\text { telephone follow-up would be } \\
\text { appropriate some of the up- }\end{array}$ & $37.5 \%$ & $62.5 \%$ \\
coming evaluations? & & \\
\hline
\end{tabular}

Table 11 Data obtained from Perceived Quality survey to patients

\begin{tabular}{llll}
\hline & \multicolumn{2}{c}{$\begin{array}{c}\text { Mean } \\
\text { deviation }\end{array}$} & Range \\
Age & 58.45 & 16.78 & $18-93$ years \\
Telematic evaluation & 8.32 & 1.24 & $3-10$ points \\
Information received & 8.01 & 1.57 & $1-10$ points \\
Sex & Female (61.9\%) & Male \\
& & $(38.1 \%)$ \\
& Yes & No \\
$\begin{array}{l}\text { 3. Did your doctor dedicated enough } \\
\text { time during your telematics }\end{array}$ & $91.4 \%$ & $8.6 \%$ \\
$\quad \begin{array}{l}\text { consultation to evaluate your case? } \\
\text { 4. Are you satisfied with telematics } \\
\text { evaluation? }\end{array}$ & $63.8 \%$ & \\
$\begin{array}{l}\text { 5. Would you prefer face-to-face } \\
\text { evaluation? }\end{array}$ & $69 \%$ & $36.2 \%$ \\
$\begin{array}{l}\text { 6. Do you think that telematics } \\
\text { follow-up would be appropriate } \\
\text { for some of your upcoming con- } \\
\text { sultations? }\end{array}$ & $49 \%$ & $31 \%$ \\
$\begin{array}{l}\text { 7. Would you prefer videoconference } \\
\text { instead of phone calls? }\end{array}$ & & $51 \%$ \\
\hline
\end{tabular}

clinics. With regard to patient satisfaction on the other hand, patient survey had better result with a mean of 8.3 points. Although we cannot rule out the potential bias in their answers given the critical situation of the lockdown, this score also proves that our patients are open minded in terms of adoption of new technologies for health care delivery in outpatient clinics.

This study has several strengths. First of all, it compares the same time period of the year in two completely different situations (pre-SARS-CoV-2 vs SARS-CoV-2); it studies an important patient series, and it assesses objective and subjective information from surgeons and patients with encouraging results. Some drawbacks should also be mentioned, as data drawn from the investigation cannot necessarily be extrapolated to a postpandemic scenario since patient and surgeon opinion could be biased by the exceptional SARS-CoV-2 pandemic.

In conclusion, this investigation provides data of an Orthopaedic and Trauma Department in a tertiary-level downtown academic hospital at the epicenter of SARSCoV-2 pandemic in Europe. Changes to highlight include a severe decrease in surgical activity, a reduction in ER attendance and delay, an increase of outpatient surgery rate, and the efficient role that new technologies and telemedicine can play in our practice. Even though the acute threat of SARS-CoV-2 will pass, things will not return completely as they were before and it is expected that this experience will set up a new paradigm for health care delivery in orthopaedics and trauma. 
Acknowledgments The authors wish to thank Jose María Luengo Alonso for his technical assistance in the analysis and editing of the manuscript.

\section{Compliance with ethical standards}

Institutional review board approval under act $07 / 20$ was obtained prior to the commencement of the study and informed consent was obtained from all patients.

Conflict of interest The authors declare that they have no conflicts of interest.

\section{References}

1. Zhu N, Zhang D, Wang W, Li X, Yang B, Song J, Zhao X, Huang B, Shi W, Lu R, Niu P, Zhan F, Ma X, Wang D, Xu W, Wu G, Gao GF, Tan W (2020) China novel coronavirus investigating and research team. A novel coronavirus from patients with pneumonia in China. N Engl J Med 382(8):727-733

2. Lu H, Stratton CW, Tang Y (2020) Outbreak of pneumonia of unknown etiology in Wuhan, China: the mystery and the miracle. J Med Virol 92(4):401-402. https://doi.org/10.1002/jmv.25678

3. Meo SA, Alhowikan AM, Al-Khlaiwi T (2020) Novel coronavirus 2019-nCoV: prevalence, biological and clinical characteristics comparison with SARS-CoV and MERS-CoV. Eur Rev Med Pharmacol Sci 24(4):2012-2019

4. Halawi MJ, Wang DD, Hunt TR (2020) What's important: weathering the COVID-19 crisis. J Bone Joint Surg 102(9):759760. https://doi.org/10.2106/JBJS.20.00419

5. Cao Y, Li Q, Chen J, Guo X, Miao C, Yang H (2020) Hospital emergency management plan during the COVID-19 epidemic. Acad Emerg Med 27(4):309-311. https://doi.org/10.1111/acem. 13951

6. Adalja AA, Toner E, Inglesby TV (2020) Priorities for the US health community responding to COVID-19. JAMA. https://doi. org/10.1001/jama.2020.3413
7. Bhatraju PK, Ghassemieh BJ, Nichols M, Kim R, Jerome KR, Nalla AK (2020) Covid-19 in critically ill patients in the Seattle region — case series. N Engl Theatr J 30:NEJMoa2004500. https:// doi.org/10.1056/NEJMoa2004500

8. Who.int. (2020). Coronavirus. [online] Accessed 2020 April 20.Available at- https-www.who.intemergenciesdiseasesnovelcoronavirus-2019

9. Spanish National Ministry of Health: Accessed 2020 April 20. https://www.mscbs.gob.es/en/profesionales/saludPublica/ccayes/ alertasActual/nCov-China/documentos/Actualizacion_82_ COVID-19.pdf

10. Coccolini F, Perrone G, Chiarugi M, Di Marzo F, Ansaloni L, Scandroglio I (2020) Surgery in COVID-19 patients: operational directives. World J Emerg Surg 15(1):1-7. https://doi.org/10.1186/ s13017-020-00307-2

11. Ti LK, Ang LS, Foong TW, Ng BSW (2020) What we do when a COVID-19 patient needs an operation: operating room preparation and guidance. Can J Anesth 6:1-3. https://doi.org/10.1007/s12630020-01617-4

12. Iacobucci G (2020) Covid-19: all non-urgent elective surgery is suspended for at least three months in England. BMJ 368:1106

13. Chang Liang Z, Wang W, Murphy D, Po Hui JH (2020) Novel coronavirus and orthopedic surgery: early experiences from Singapore. J Bone Joint Surg 20:745-749. https://doi.org/10. 2106/JBJS.20.00236

14. Mavrogenis AF, Quaile A, Scarlat MM (2020) The virus crisis affects Orthopaedic surgery and scientific activities worldwide. Int Orthop Apr 44(5):813-817. https://doi.org/10.1007/s00264020-04557-2

15. Von Elm E, Altman DG, Egger M (2007) The strengthening the reporting of observational studies in epidemiology (STROBE) statement: guidelines for reporting observational studies. Lancet 370:1453-1457. https://doi.org/10.1016/S0140-6736(07)61602-X

Publisher's note Springer Nature remains neutral with regard to jurisdictional claims in published maps and institutional affiliations. 